\title{
Chromatic Dispersion Measurement in Graded-Index Multimode Optical Fibers
}

\author{
Gair D. Brown
}

\begin{abstract}
The chromatic dispersion observed in a graded-index multimode optical fiber is a function of the radial position of the launched light. When a graded-index multimode optical fiber is overfilled spatially and angularly, the chromatic dispersion observed is an average (composite) chromatic dispersion over all of the possible launches. Under certain assumptions, the relationship between the chromatic dispersion observed for the overfilled fiber and the chromatic dispersion of any restrictive launch can be determined. This relationship can be used to predict the composite graded-index multimode optical fiber chromatic dispersion from the measurement of the chromatic dispersion of a single simplified launch, such as a single-mode fiber launch. Preliminary experimental results confirm this approach.
\end{abstract}

\section{INTRODUCTION}

$\mathbf{T}$ THE measurement of the composite chromatic dispersion of a graded-index multimode optical fiber is nontrivial. The chromatic dispersion of a graded-index multimode fiber is defined as the composite chromatic dispersion observed using a light source that uniformly overfills the fiber [1]. The use of the uniformly overfilled launch, however, introduces modal distortion into the test signal. Setting up uniform overfill light launch conditions and removing the effects of modal distortion from the data is difficult. Moreover, the results obtained from the measurement generally are not as precise as those for single-mode fiber because of modal distortion.

One solution to this problem is to define a chromatic dispersion measurement condition for the multimode optical fiber which is easy to implement, highly repeatable, and which can be easily correlated to the composite optical fiber chromatic dispersion. A simplified launch condition for the measurement is an extremely low-order mode launch in which only a few modes of the multimode optical fiber are excited. A low-order launch can be initiated in the graded-index multimode optical fiber using a single-mode optical fiber as the light source. In this paper, the relationship between the chromatic dispersion for an extremely low-order launch and the composite chromatic dispersion of the optical fiber is investigated.

\section{LOCAL Chromatic DisPersion}

The local chromatic dispersion, the chromatic dispersion associated with the particular glass composition at any radial

Manuscript received February 23, 1993; revised August 3, 1994. This work was supported by the Naval Sea Systems Command Fiber Optic Program Office.

The author is with the Fiber Optics Group, Naval Surface Warfare Center, Dahlgren, VA 22448-5000 USA.

IEEE Log Number 9405766. position in the fiber, can be determined from the index of refraction of the glass composition at that position. If the index of refraction as a function of wavelength $\lambda$ is given by a 3-term Sellmeier equation

$$
n(\lambda)=A \lambda^{2}+B-C \lambda^{-2}
$$

where $A, B$, and $C$ are constants for a particular glass composition, the dispersion $D$ and the dispersion slope, $S$ are then

$$
\begin{aligned}
D & =\frac{d}{c d \lambda}\left(n-\lambda \frac{d n}{d \lambda}\right) \\
& =\frac{1}{C}\left(-2 A \lambda+6 C \lambda^{-3}\right)
\end{aligned}
$$

and

$$
S=\frac{d D}{d \lambda}=-\frac{1}{C}\left(2 A+18 C \lambda^{-4}\right)
$$

where $c$ is the speed of light in vacuum. From (2) and (3), the zero-dispersion wavelength $\lambda_{o}$ and the dispersion slope at the zero-dispersion wavelength $S_{o}$ can be shown to be

$$
\begin{aligned}
\lambda_{0} & =\sqrt[4]{3 \frac{C}{A}} \\
S_{0} & =-8 \frac{A}{C} .
\end{aligned}
$$

If two glasses are nearly identical but differ slightly in the concentrations of some dopant, we can assume that the functional dependence of the index of refraction with wavelength is nearly identical for the two materials. However, the wavelength-independent portion of the index of refraction is changed by an amount $\delta B$ and wavelengths in the lower index of refraction material are mapped to higher wavelengths in the higher index of refraction material by an amount $\delta \lambda$.

If this is applied to a graded-index multimode fiber and the index of refraction at the center of the fiber is given by (1), then the index of refraction at any radial position, $n_{r}(\lambda)$, is given by

$$
\begin{aligned}
n_{r}(\lambda)=A_{o} \lambda^{2}\left(1+\frac{\delta \lambda_{r}}{\lambda}\right)^{2}+B_{o} & -\delta B_{r} \\
& +C_{o} \lambda^{-2}\left(1+\frac{\delta \lambda_{r}}{\lambda}\right)^{-2}
\end{aligned}
$$

where $A_{o}, B_{o}$, and $C_{o}$ are the Sellmeier coefficients for the glass at the center of the fiber, and $\delta B_{r}$ and $\delta \lambda_{r}$ are adjustments to $B$ and $\lambda$ for the radial position $r$. 
As long as $\delta \lambda_{r} \ll \lambda$ and the wavelengths of interest are within a small range around some center wavelength, then

$$
\frac{\delta \lambda_{r}}{\lambda} \approx \delta_{r}
$$

and the correction terms are approximately independent of wavelength. If the wavelengths of interest range around $\lambda=$ $\lambda_{o}$, the zero-dispersion wavelength $\lambda_{o r}$ and the dispersion slope at the zero-dispersion wavelength $S_{o r}$ at each radial position can be expressed as

$$
\begin{aligned}
\lambda_{o r}^{4} & =\frac{3 C}{A\left(1+\delta_{r}\right)^{4}}=\frac{\lambda_{o o}^{4}}{\left(1+\delta_{r}\right)^{4}} \\
S_{o r} & =\frac{-8 A\left(1+\delta_{r}\right)^{2}}{C}=S_{o o}\left(1+\delta_{r}\right)^{2}
\end{aligned}
$$

where $\lambda_{o o}$ and $S_{o o}$ are defined at the center of the fiber, and the other terms are defined as before. $\delta_{r}$ is found by investigating the function

$$
F_{r}=\sqrt{n_{o}^{2}-n_{r}^{2}}=\sqrt{\left(n_{o}+n_{r}\right)\left(n_{o}-n_{r}\right)}=N A \sqrt{f(r)}
$$

where at the center of the fiber

$$
n_{o}(\lambda)=A_{o} \lambda^{2}+B_{o}+C_{o} \lambda^{-2}
$$

$n_{r}$ is given by (6), NA is the fiber numerical aperture, and $f(r)$ is defined from the fiber's refractive index profile $(R I P)$ by

$$
R I P=n_{o}\left[1-\left(\frac{n_{o}^{2}-n_{c}^{2}}{n_{o}^{2}}\right) f(r)\right]^{1 / 2}
$$

where $n_{c}$ is the index of refraction of the fiber cladding material. If the expressions for $n_{o}$ and $n_{r}$ are substituted into (9), and $\delta_{r}$ is assumed to be much less than $1, \delta_{r}$ can be expressed as

$$
\delta_{r}=\frac{N A^{2} f(r)-\left(n_{o}+n_{c}\right) \delta B_{r}}{2\left(n_{o}+n_{c}\right) \lambda^{2}\left[\frac{S_{o} c}{8}+\frac{\lambda_{o}^{4} S_{o} c}{24 \lambda^{2}}\right]}
$$

where the variables are defined as before.

$\delta B_{r}$ varies from a minimum value of zero at the center of the fiber to a maximum value of $\delta B_{c}$ at the fiber corecladding interface. To the first order, it is expected that $\delta B_{r}$ would behave similarly to $\delta n=n_{o}-n_{r}$. The variation of $\delta n$ with respect to $r$ is approximately given by

$$
\delta n=\left(n_{o}-n_{c}\right) f(r)
$$

so that

$$
\delta B_{r}=\delta B_{c} f(r)
$$

Substituting for $\delta B_{r}$ in (13) then leads to

$$
\delta_{r}=\frac{N A^{2}-\left(n_{o}+n_{c}\right) \delta B_{c}}{2\left(n_{o}-n_{c}\right) \lambda^{2}\left[\frac{S_{o} c}{8}+\frac{\lambda_{o}^{4} S_{o} c}{24 \lambda^{2}}\right]} f(r)=\delta_{c} f(r) .
$$

From (7) $\delta_{c}$ is given by $\delta \lambda_{c} / \lambda$ so $\delta_{r}$ can be most simply expressed as

$$
\delta_{r}=\frac{\delta \lambda_{c}}{\lambda_{o}} f(r)
$$

If the cladding material is pure silica and the $R I P$ is approximately parabolic, the zero-dispersion wavelength of the cladding material is approximately $1273 \mathrm{~nm}$, and $f(r)$ is given by

$$
f(r)=\left(\frac{r}{a}\right)^{g}
$$

where $a$ is the fiber core radius and $g$ is the profile parameter. The zero-dispersion wavelength and the dispersion slope at the zero-dispersion wavelength at any radial position $r$ are then given by

$$
\begin{aligned}
\lambda_{o r} & =\frac{\lambda_{o o}}{\left[1+\frac{\left(\lambda_{o o}-1273\right)}{\lambda_{o o}}\left(\frac{r}{a}\right)^{g}\right]} \\
S_{o r} & =S_{o o}\left[1+\frac{\left(\lambda_{o o}-1273\right)}{\lambda_{o o}}\left(\frac{r}{a}\right)^{g}\right]^{2}
\end{aligned}
$$

where all wavelengths are expressed in nanometers.

\section{COMPOSITE CHROMATIC DISPERSION}

To find the composite $\lambda_{o}$ and $S_{o}$ of a uniformly overfilled multimode optical fiber, the local chromatic dispersion at each radial position is weighted by the fraction of the total power carried at that radial position. The composites $\lambda_{o}$ and $S_{o}$ are calculated from

$$
\begin{aligned}
\lambda_{o} & =\frac{\int_{0}^{a} P(r) \lambda_{o}(r) d r}{\int_{0}^{a} P(r) d r} \\
S_{o} & =\frac{\int_{0}^{a} P(r) S_{o}(r) d r}{\int_{0}^{a} P(r) d r}
\end{aligned}
$$

which, when evaluated, become

$$
\begin{aligned}
& \lambda_{o}=\lambda_{o o}\left[1-\frac{\delta_{c}}{g+1}\right] \\
& S_{o}=S_{o o}\left[1+\frac{2 \delta_{c}}{g+1}\right] .
\end{aligned}
$$

\section{RESULTS}

The chromatic dispersions of three multimode graded-index fibers were measured. Fibers 1 and 2 were measured by Corning, Inc. and the Naval Surface Warfare Center, and fiber 3 was measured entirely by York Technology, Inc. [2]. Each fiber was measured using both a uniform overfill launch and a restricted launch implemented with a single-mode fiber. The values obtained using the single-mode launch were used in (23) and (24) to obtain estimations of the total multimode fiber chromatic dispersion. The results are summarized in Table I.

\section{CONCLUSIONS}

The preliminary results show excellent correlation between the composite chromatic dispersion (including zero-dispersion wavelength and dispersion slope) calculated from a singlemode fiber launch and that measured directly using overfill 
TABLE I

PreliminaRY Results

\begin{tabular}{|c|c|c|c|c|c|c|}
\hline$\underset{\#}{\text { Eiber }}$ & $\begin{array}{c}\lambda_{\infty o} \\
\text { (meas, } \\
\text { nm) }\end{array}$ & $\begin{array}{c}\lambda_{\circ} \\
\text { (meas, } \\
\text { nm) }\end{array}$ & $\begin{array}{c}\lambda_{0} \\
(\mathrm{calc}, \\
\mathrm{nm})\end{array}$ & $\begin{array}{c}\mathrm{S}_{\infty} \\
\text { (meas, } \\
\mathrm{ps} / \mathrm{nm}^{2} \mathrm{~km} \text { ) }\end{array}$ & $\begin{array}{c}\mathrm{S}_{0} \\
\text { (meas, } \\
\mathrm{ps} / \mathrm{nm}^{2} \mathrm{~km} \text { ) }\end{array}$ & $\begin{array}{c}\mathrm{S}_{\mathrm{o}} \\
\text { (calc, } \\
\mathrm{ps} / \mathrm{nm}^{2} \mathrm{~km} \\
\text { ) }\end{array}$ \\
\hline 1 & 1373 & 1342 & 1341 & 0.0931 & 0.0945 & 0.0975 \\
\hline 2 & 1330 & 1313 & 1312 & 0.0984 & 0.0957 & 0.1011 \\
\hline 3 & 1332 & 1315 & 1313 & 0.0871 & 0.0896 & 0.0896 \\
\hline
\end{tabular}

launch conditions. Therefore, the composite chromatic dispersion of a graded-index multimode optical fiber can be calculated from chromatic dispersion measurements at the center of the multimode fiber. Since the chromatic dispersion measurement at the center of a graded-index multimode optical fiber can be implemented using a single-mode fiber launch, graded-index multimode optical fiber chromatic dispersion measurements can be taken without the effects of modal distortion.

\section{ACKNOWLEDGMENT}

The author would like to thank Dr. A. Barlow and J. Parton for their helpful discussions regarding this work. The author would like to thank D. Thomas of the Naval Surface Warfare Center, and the Corning, Inc., Waveguide Product Engineering Lab for their assistance in making chromatic dispersion measurements on fibers 1 and 2 .

\section{REFERENCES}

[1] TIA/EIA-455-168, "Chromatic dispersion measurement of multimode graded-index and single-mode optical fibers by spectral group delay measurement in the time domain."

[2] Meet. Minutes, Electron. Ind. Assoc. Telecommun. Ind. Assoc., FO6.6.1, Apr. 1991.

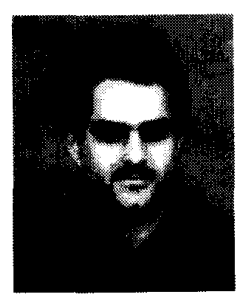

Gair D. Brown received the B.S. degree in physics and mathematics (summa cum laude) from West Virginia Institute of Technology in May 1985.

He has worked in the Fiber Optics Group at the Naval Surface Warfare Center, Dahlgren, since June 1985 , concentrating on shipboard cable plant design, specifications and standards, and multimode and single-mode fiber-optic systems design. 